\title{
Heart block and nonocclusive mesenteric ischemia
}

\author{
Christopher W. Baugh • Adam C. Levine • \\ Daniel J. Pallin
}

Received: 13 January 2009 / Accepted: 22 February 2009 /Published online: 29 April 2009

(C) Springer-Verlag London Ltd 2009

An 88-year-old woman presented with 1 day of lower abdominal pain and bloody diarrhea. She claimed fatigue and dyspnea on exertion for the past 3 weeks. She had been healthy all her life and took no medications. She was afebrile with a heart rate of 40 beats per minute and blood pressure of 134/61 mmHg. Her abdomen was soft with minimal lower tenderness, and the exam was otherwise normal. Lab chemistries, lactate, and $\mathrm{CBC}$ were normal except WBC of 23.54. We obtained the ECG shown in Fig. 1 and the abdominal CT shown in Fig. 2. She was admitted to the cardiology service. We ordered the CT because we believed that any abdominal tenderness in such

\author{
C. W. Baugh $\cdot$ A. C. Levine $\cdot$ D. J. Pallin \\ Department of Emergency Medicine, \\ Brigham and Women's Hospital, \\ Boston, MA, USA \\ A. C. Levine \\ e-mail: alevine2@partners.org \\ D. J. Pallin \\ e-mail: dpallin@partners.org \\ C. W. Baugh · A. C. Levine \\ Harvard-Affiliated Emergency Medicine Residency, \\ Boston, MA, USA \\ D. J. Pallin \\ Division of Emergency Medicine, Children's Hospital Boston, \\ Boston, MA, USA \\ C. W. Baugh $(\bowtie)$ \\ 1163 Beacon Street \#4, \\ Brookline, MA 02446, USA \\ e-mail: cbaugh@partners.org
}

an elderly patient could indicate serious pathology, such as abscess, aortic aneurysm, or mesenteric ischemia.

Upon further evaluation, she was found to have heart block with non-occlusive mesenteric ischemia. The ECG reveals second-degree atrioventricular block with 2:1 conduction. If the PR interval increases progressively prior to a non-conducted $\mathrm{P}$ wave, the atrioventricular block is Mobitz type I. In Mobitz type II, the nonconducted beats are not heralded by an increasing PR interval, as noted in this patient [1]. All type II or symptomatic type 1 patients are candidates for pacemaker implantation [2].

The CT image reveals colonic wall thickening with mesenteric stranding, consistent with ischemic colitis. However, the mesenteric vessels were patent, leading to a diagnosis of nonocclusive mesenteric ischemia. Up to $30 \%$ of mesenteric ischemia cases fall into this category, usually in the setting of low cardiac output [3]. Previous case reports most often describe this phenomenon in settings such as hemodialysis and cardiac or vascular surgery [4-6]. As with all mesenteric ischemia, the classic presentation is pain out of proportion to the exam; hematochezia and lactic acidosis are late findings whose absence should not influence the workup. We believe our patient's heart block and bradycardia impaired cardiac output, causing bowel hypoperfusion. The normal blood pressure we observed must have belied prior periods of hypotension.

Treatment is directed at reversal of the low-flow state, sometimes followed by injection of vasodilators such as papaverine into the mesenteric vessels [3]. Our patient had a pacemaker placed and subsequently recovered uneventfully. 


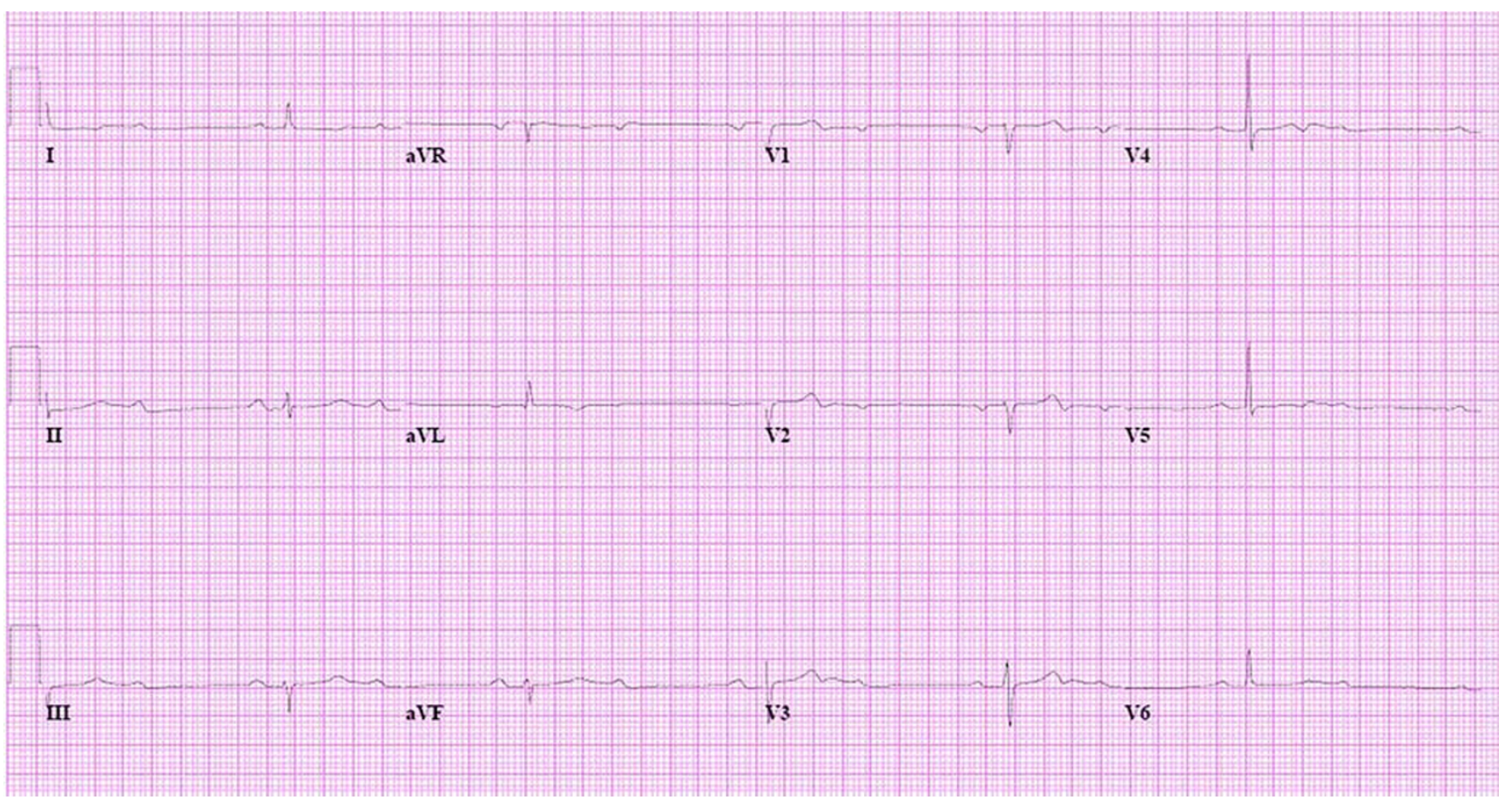

Fig. 1 Electrocardiogram showing regular bradycardia with a 2:1 ratio of $\mathrm{P}$ waves to QRS complexes

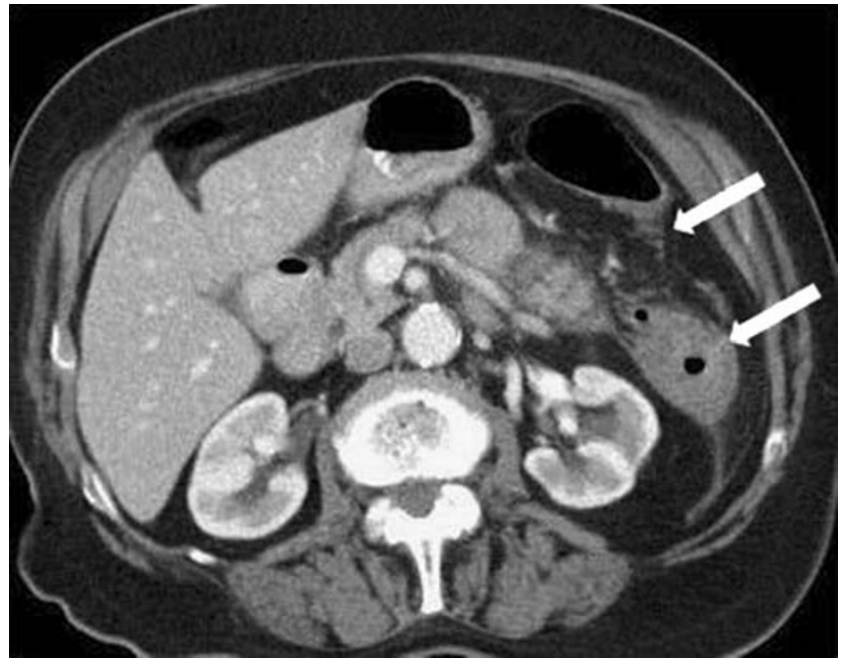

Fig. 2 Transverse computed tomographic (CT) image showing colonic wall thickening and surrounding mesenteric stranding (arrows)

\section{References}

1. Mangrum JM, DiMarco JP (2000) The evaluation and management of bradycardia. NEJM 342(10):703-9

2. Barold SS, Hayes DL (2001) Second degree atrioventricular block: a reappraisal. Mayo Clin Proc 76:44-57

3. Trompeter M, Brazda T, Remy CT et al (2002) Non-occlusive mesenteric ischemia: etiology, diagnosis, andinterventional therapy. Eur Radiol 12(9):1179-1187

4. John AS, Tuerff SD, Kerstein MD (2000) Nonocclusive mesenteric infarction in hemodialysis patients. Journal of the American College of Surgeons 190(1):84-88

5. Klotz S, Vestring T, Rotker J et al (2001) Diagnosis and treatment of nonocclusive mesenteric ischemia after open heart surgery. Annals of Thoracic Surgery 72(5):1583-1586

6. Kadono J, Hamada N, Ishizaki N et al (2002) Recurrent nonocclusive mesenteric ischemia after resection of iliac artery aneurysm.[see comment]. Journal of Gastroenterology 37(2):123128 